\title{
INVESTIGACIONES
}

\section{La pedagogía del inglés para refugiados. Proyecto de vinculación universitaria}

\author{
English pedagogy for refugees: A project of University outreach
}

\author{
Haydée Ramírez, Ingrid Valentina Grijalva Arriaga ${ }^{b}$ \\ ${ }^{a}$ Escuela de Lingüística Aplicada, Pontificia Universidad Católica de Ecuador, Sede Esmeraldas. \\ Correo electronico: haydee.ramirez@pucese.edu.ec \\ ${ }^{\mathrm{b}}$ Escuela de Lingüística Aplicada, Pontificia Universidad Católica de Ecuador, Sede Esmeraldas. \\ Correo electronico: Ingrid.grijalva@ pucese.edu.ec
}

\begin{abstract}
Se analiza el proceso pedagógico del inglés para la sostenibilidad de refugiados como parte de un programa de vinculación de la universidad con la comunidad. El análisis se enmarca en la Enseñanza del inglés con Fines Específicos, el Enfoque Comunicativo para el Aprendizaje de Lenguas, la Teoría Humanística, el enfoque Histórico-Cultural de Vygotsky y la Pedagogía Liberadora. Se utilizó una población de 15 estudiantes refugiados que asistieron a clases de inglés en el período de abril a septiembre de 2016 y cinco estudiantes de la Escuela de Lingüística Aplicada que trabajaron como docentes de inglés. Se utilizaron los métodos hermenéutico-dialéctico, sistémico- estructural- funcional, analítico-sintético y manual porcentual, en un enfoque de investigación mixto (cuantitativo-cualitativo). Los resultados revelaron la necesidad de organizar el proceso pedagógico del inglés a refugiados para que estos logren su reasentamiento e inserción en la sociedad.
\end{abstract}

Palabras clave: proceso pedagógico, sostenibilidad, metodología, fines específicos.

\begin{abstract}
The pedagogical process of English for the sustainability of refugees, as part of a program of outreach linking the university with the community, was analyzed. The analysis is centered on the teaching of English for Specific Purposes, the Communicative Approach to language teaching, the Humanistic Theory, the Historical-Cultural Approach by Lev Vygotsky, and Liberating Pedagogy. A population of 15 student-refugees who attended English lessons in the period from April to September 2016 was studied, as well as 5 students from the School of Applied Linguistics who worked as English teachers. The hermeneutic-dialectic, structural-functional-systemic, analytic-synthetic, and manual percentage methods were used in a mixed methodological approach (quantitativequalitative). The results revealed the necessity to organize the pedagogical process of English for refugees so that they can achieve their resettlement and integration into society.
\end{abstract}

Key words: pedagogical process, sustainability, methodology, occupational purposes. 


\section{INTRODUCCIÓN}

En los estatutos de las Naciones Unidas se han direccionado objetivos encaminados a poner fin a la pobreza y las desigualdades, promoviendo sociedades pacíficas e inclusivas, que brinden protección al hombre, de manera genérica, y todos sus derechos. Para lograr este empeño, la educación constituye un eje crucial. Como apunta el documento oficial, (Asamblea General de las Naciones Unidas, 2015), entre otros objetivos:

Estamos resueltos a poner fin a la pobreza y el hambre en todo el mundo de aquí a 2030, a combatir las desigualdades dentro de los países y entre ellos, a construir sociedades pacíficas, justas e inclusivas, a proteger los derechos humanos y promover la igualdad entre los géneros y el empoderamiento de las mujeres y las niñas, y a garantizar una protección duradera del planeta y sus recursos naturales. Estamos resueltos también a crear las condiciones necesarias para un crecimiento económico sostenible, inclusivo y sostenido, una prosperidad compartida y el trabajo decente para todos, teniendo en cuenta los diferentes niveles nacionales de desarrollo y capacidad...Al emprender juntos este gran viaje, prometemos que nadie se quedará atrás(2015: 3).

En los entornos urbanos, existe una estrecha relación entre la protección y los medios de vida sostenibles. Las personas desplazadas forzosamente tienen una apremiante necesidad de adquirir bienes y servicios y dinero en efectivo todos los días, pero muchos aspectos de los contextos urbanos hacen que la búsqueda de los medios de vida sostenibles sea un riesgo. Al ayudar a estas personas a encontrar medios de vida sostenibles, también se les ayuda a restaurar su dignidad e independencia. Es por ello que constituye una urgencia el promover y garantizar el derecho de los refugiados a tener acceso al trabajo y otras formas de vida sostenibles.

Como respuesta a esta demanda, y dando cumplimiento a las tres aristas fundamentales en la Universidad del Siglo XXI: docencia, investigación y vinculación con la sociedad, nuestra alta casa de estudios desarrolla el proyecto de vinculación Fortalecimiento de medios de vida sostenibles e integración local, con ACNUR como institución aliada. La Escuela de Lingüística Aplicada participa del mismo en la enseñanza del inglés a los refugiados, con un docente responsable que está a cargo de la guía, supervisión y control de las actividades, y un grupo de estudiantes de la especialidad que son asignados para diseñar las clases y materiales didácticos e impartir la docencia.

Los estudiantes refugiados son hablantes hispanos. Hay un grupo de ellos que son trasladados a países de habla inglesa para su reasentamiento; pero las carencias lingüísticas en este idioma afectan sustancialmente su integración a la sociedad. Otros refugiados, sin embargo, se han reasentado y establecido en la provincia y son emprendedores. Debido al hecho de que en esta región hay una elevada afluencia de turistas del área internacional, el idioma inglés se convierte en vital para la comunicación y desarrollo cultural de ambos grupos.

Constituye una necesidad atender tanto el proceso de enseñanza-aprendizaje como de educación de estos estudiantes refugiados. Generalmente, los cursos se imparten basados en Inglés General, sin atender sus necesidades específicas de comunicación y sus particularidades como estudiantes que viven en otro contexto cultural y tienen temores y frustraciones por antecedentes de violencia, agresión, persecución, entre otros. La 
experiencia en el trabajo con este proyecto ha permitido revelar carencias en la organización del proceso pedagógico del inglés para estos estudiantes, que les permita desarrollar su integración social y sostenibilidad. Esto conduce a plantear como pregunta de investigación: ¿Cómo implementar el proceso pedagógico de inglés para estudiantes refugiados?

Como objetivo general nos propusimos analizar el proceso pedagógico del inglés para refugiados. Como objetivos específicos, determinar las teorías pedagógicas y de la didáctica del inglés que respaldan este estudio; diagnosticar las necesidades de aprendizaje de los mencionados estudiantes; y proponer una metodología sustentada en un modelo pedagógico que contribuya a la preparación idiomática en inglés de estos estudiantes, que les permita su inclusión social y sostenibilidad.

\section{ENSEÑANZA DEL INGLÉS CON FINES ESPECÍFICOS Y DIAGNÓSTICO DE NECESIDADES DE APRENDIZAJE}

Para llevar a cabo esta investigación, se ha tenido en cuenta la Enseñanza del idioma inglés con Fines Específicos (IFE), que de acuerdo a Hutchinson y Waters (1994) se refiere a que los contenidos y métodos a enseñar están determinados por las necesidades de aprendizaje de los estudiantes. Esta definición es entendida como la necesidad de realizar un diagnóstico a los estudiantes refugiados para determinar qué necesitan aprender en idioma inglés para cubrir sus expectativas de comunicación.

Teniendo en cuenta que los refugiados buscan elementos de la lengua para comunicarse dentro de los términos de sus oficios, se decide dirigir la enseñanza del inglés a fines ocupacionales (IFO). Esto implica primeramente conocer qué ocupaciones desempeñan los estudiantes para luego diseñar los contenidos a enseñar: léxico, funciones comunicativas, de acuerdo a las mismas.

Para el análisis de las necesidades de aprendizaje de los estudiantes, se deben tener en cuenta diversos elementos planteados por Hutchinson y Waters (1994):

¿Por qué los estudiantes van a tomar el curso? Si es opcional u obligatorio; si hay una necesidad aparente o no, si el status, el dinero o la promoción están involucrados; qué esperan obtener los estudiantes; cuál es su actitud hacia el curso; si desean mejorar su inglés o simplemente pasar el tiempo.

¿Cómo aprenden los estudiantes? Cuál es su experiencia de aprendizaje; qué concepto tienen de enseñanza-aprendizaje; qué metodología los beneficiaría; qué tipo de técnicas serían adecuadas.

¿Qué recursos están disponibles? El número y la competencia de los docentes; la actitud de los docentes hacia el curso de IFE, en este caso IFO; el conocimiento de los docentes y su actitud con relación al contenido; materiales; oportunidades para realizar actividades fuera del aula.

¿Quiénes son los estudiantes? Edad, sexo, nacionalidad; qué ya saben acerca del inglés; qué contenido de aprendizaje ya conocen; cuáles son sus intereses; cuál es su entorno socio-cultural; a qué estilos de aprendizaje están acostumbrados; cuál es su actitud hacia el inglés o hacia la cultura de los hablantes nativos del inglés. ¿Dónde va a tener lugar el curso de IFE? Cómo son los alrededores del aula, si son agradables, bulliciosos, fríos, etc. 
¿Cuándo va a tener lugar el curso de IFE? Hora del día; si es todos los días o una vez por semana; si es a tiempo completo o parcial; concurrente a las necesidades o con un diagnóstico previo (1994: 62-63).

Se necesita, además, hacer una revisión de las metodologías más avanzadas para enseñar el idioma inglés.

\section{MÉTODOS Y TEORÍAS QUE DETERMINAN LA PEDAGOGÍA DEL INGLÉS}

Con relación a la metodología para la enseñanza del idioma inglés, el Enfoque Comunicativo permite al estudiante refugiado desarrollar el aprendizaje del idioma teniendo en cuenta las cuatro principales destrezas: escuchar, hablar, leer y escribir. Esta combinación de habilidades comunicativas (Richards y Rodgers, 1986) es crucial para el dominio de la lengua extranjera. Precisamente, Hymes (1970) establece que el objetivo para enseñar idiomas estriba en el desarrollo de la competencia comunicativa.

Para lograr individuos competentes en una lengua, el uso de la Teoría Humanística se convierte en un elemento primordial. Como apuntan Williams y Burden (2005), citando a Rogers, existen elementos importantes para brindar un enfoque humanístico a la educación. Primeramente, se refieren al hecho de que los seres humanos tienen un gran potencial para el aprendizaje, lo cual implica que un aprendizaje será significativo si lo que se imparte se entiende como importante por la persona que aprende. Esto implica que es necesario involucrar los sentimientos del aprendiz en su aprendizaje, lo que significa tener en cuenta las dimensiones cognitiva y afectiva.

Por lo anterior, es imprescindible hacer un diagnóstico de las necesidades de aprendizaje de los estudiantes para diseñar las actividades docentes. La respuesta a qué necesita el estudiante aprender, enfática en la enseñanza del inglés con fines específicos, muestra cómo este tipo de enseñanza es humanística.

El creer en el hombre como ser humano, insta también a los educadores a tener en cuenta la Teoría Liberadora de Freire (s.f) en la clase de inglés. Resulta crucial rechazar la idea de que las personas permanecen ignorantes sin aprender la cultura de los poderosos, porque cada ciudadano es un portador de la cultura con su visión personal de la realidad. Hay una esperanza a través de la pedagogía, cada cual puede expresarse libremente sin temor a ser censurado de manera negativa.

De igual manera, Hooks (2003) enfatiza la importancia de promover aprendizajes entre los estudiantes y entre estos y los docentes, de manera que ningún docente tenga el poder de usar el aula con dominio. La idea es de crear un ambiente fraterno y de libertad, donde se puedan expresar los sentimientos y donde haya un respeto hacia el estudiante. Según el autor mencionado, el aula debe ser un lugar para expandir la mente de los estudiantes, en un trabajo colaborativo estudiantes-estudiantes y estudiantes-docentes.

Los estudiantes refugiados deben sentirse fraternalmente libres para hablar en inglés sobre sus vivencias, sobre su familia, sus comidas, música y otros elementos culturales. El aprender inglés como lengua internacional de comunicación para preparase para la sostenibilidad en el reasentamiento, no quiere decir que deben olvidar su cultura, más bien apropiarse de ella, defenderla y aprender la de los hablantes del inglés como elemento enriquecedor de su propia cultura, ambas reteniendo su valía. 
La necesidad de lograr un aprendizaje en el contexto social, en el intercambio de experiencias, le da una posición preponderante al enfoque Histórico-Cultural en este estudio. Domínguez (2013) citando a Vygotsky enfatiza el papel de la sociedad en la formación de los procesos psíquicos y de la personalidad del individuo.

El enfoque mencionado define claramente dos niveles evolutivos en el proceso de aprendizaje del estudiante. Un nivel relacionado a las capacidades reales de aprender y otro relacionado a las posibilidades de aprender con la ayuda de otros estudiantes: Zona de Desarrollo Actual y Zona de Desarrollo Próximo. Esta última caracteriza el desarrollo perspectivo del individuo por promover, en cada momento del aprendizaje, una nueva zona de desarrollo, la cual niega dialécticamente a la que le antecede con la apropiación y aplicación de nuevas formas que le permiten trabajar y buscar por sí solo el conocimiento, preparándolo a la vez para su auto aprendizaje.

\section{ESTUDIOS PREVIOS}

Varias investigaciones han mostrado la necesidad emergente de atender la pedagogía y la didáctica del idioma inglés para desarrollar la comunicación en este idioma por parte de refugiados y así mejorar su supervivencia en contextos de reasentamiento.

Gass (1997), refiriéndose a esta necesidad, reveló la importancia de la vinculación de la escuela y la familia, y mantener al padre informado de los progresos del estudiante. Los resultados coinciden en la vinculación necesaria de la escuela con la familia de los estudiantes, el estudio individual y trabajo independiente de los estudiantes en sus casas.

Adkins, Birman y Sample (1999) enfatizaron la relevancia que tiene para el docente de estudiantes refugiados el hecho de que se trata no solo de enseñar el idioma, sino que se debe contribuir a destruir las barreras culturales, de esta manera se ayudan a los estudiantes a ajustarse a la nueva vida y por lo tanto, de obtener salud mental a través de la disminución del estrés. Como funciones comunicativas imprescindibles para enseñar sugirieron ir de compras de alimentos y dar direcciones. Además, estos autores revelaron la conveniencia de crear materiales didácticos para el aprendizaje de la lengua, de acuerdo a lo que realmente es indispensable para el estudiante. Plantearon, además, que se debe contribuir a la salud mental de los estudiantes refugiados propiciando discusiones de interés como: ir al médico, buscar un lugar adecuado para vivir, comprar alimentos y bebidas, interaccionando con la escuela.

Hernández y Villalba (2005), citando a Cabré, encontraron en sus investigaciones que con el trabajo con inmigrantes se trata de lograr una competencia comunicativa en temas de la cotidianeidad para sobrevivir. Para ello, se sugiere el diseño de programas de capacitación que preparen a los estudiantes para el mundo laboral utilizando técnicas de búsqueda de empleo, preparación de entrevistas de trabajo, elaboración de un currículum vitae, entre otras. Se recomienda que algunos programas se realicen en las aulas y otros que propicien la enseñanza en el propio puesto de trabajo, para brindar capacitación laboral y lingüística. Por lo anterior, hay que saber determinar las tareas de aprendizaje. Estos autores apoyan el diseño de cursos relacionados con ocupaciones que no comúnmente se trabajan, como jardinería, hotelería, construcción, servicio doméstico, etc.

Munthe (2005) reveló, en sus estudios, cómo la agencia del docente y su administración pueden dar apoyo a los aprendices del idioma. Para ello el docente de inglés necesita de 
orientaciones metodológicas. Kaur (2011), por otro lado, mostró la mala concentración de los estudiantes refugiados en las clases, por lo que no recuerdan los contenidos con facilidad, de ahí que sugiere la implementación de fases de repaso de contenidos a través de toda la lección, un constante reciclaje de los contenidos, así como el uso de una variedad de materiales didácticos.

De manera similar, Black (2013) aportó los esfuerzos del docente de inglés al enseñar a estudiantes que aún no han sido alfabetizados en su lengua madre y ofreció una guía para llevar a cabo la tarea docente.

\section{METODOLOGÍA}

Tipo de investigación:

Se desarrolló una investigación de campo, cualitativa-cuantitativa, descriptivalongitudinal proyectiva.

Método:

Se utilizó el método hermenéutico dialéctico, que posibilitó la interpretación de los datos teóricos y de los resultados de las técnicas aplicadas; el método sistémico-estructuralfuncional que posibilitó el análisis de los contenidos y el diseño de la metodología; el método analítico-sintético que permitió el análisis de todo el proceso, desde la revisión de teorías, estudios previos, hasta la situación del aula y las particularidades del proceso de enseñanza-aprendizaje; y el método manual porcentual para la recolección y procesamiento de los datos.

Muestra:

La muestra coincidió con una población de 15 estudiantes refugiados que asistieron a clases de inglés de abril a septiembre del 2016, como parte del proyecto de vinculación universitario con la comunidad denominado: Fortalecimiento de medios de vida sostenibles e integración local. Además se utilizaron 5 estudiantes de la Escuela de Lingüística Aplicada que trabajaron como docentes e impartieron clases de inglés a los estudiantes refugiados. A estos últimos se les llamó estudiantes-docentes.

Técnicas:

Entrevista, observación y encuesta.

Procedimientos:

Se entrevistaron los 5 estudiantes de la Escuela de Lingüística Aplicada que realizaron la labor de docentes en el proyecto.

Se realizaron 5 sesiones de observaciones a las clases de inglés para estudiantes refugiados por parte de la docente responsable del proyecto de vinculación durante el período de abril a septiembre del 2016. Los elementos observados se anotaron en el diario de investigación y luego se analizaron.

Se encuestaron los 15 estudiantes refugiados que formaban parte de dos grupos de inglés.

Los datos obtenidos se llevaron a figuras utilizando el programa Microsoft Excel. 


\section{RESULTADOS Y DISCUSIÓN}

Los resultados de la entrevista realizada a los estudiantes de la Escuela de Lingüística que impartieron clases de inglés a refugiados revelaron lo siguiente:

- Los estudiantes refugiados se ausentan frecuentemente a clases, en mayor medida los del grupo de reasentamiento en un tercer país, lo que se piensa sea causado porque ellos son convocados para el curso en su preparación para el reasentamiento en países de habla inglesa. Es decir, ellos no eligen estar en el curso. Estos estudiantes no parecen tener conciencia absoluta de la necesidad que tienen de aprender el idioma inglés para su sostenibilidad en el lugar de reasentamiento, en el que deben solicitar trabajo, comprar, vender, ir al médico, etc., en idioma inglés. Esta actitud también puede ser causada por su estrés como refugiados en proceso de reasentamiento.

- Los estudiantes refugiados que son emprendedores muestran mayor interés por el aprendizaje del inglés, demostrado al asistir con más frecuencia a clases y hacerlo de manera voluntaria. Estos parecen tener conciencia de la necesidad del inglés para desarrollar su emprendimiento, lo que puede ser causado porque ya están asentados hace varios años, poseen sus negocios y no tienen planes de viajar.

- Los estudiantes-docentes carecen de materiales didácticos que les sirvan a los estudiantes refugiados para su aprendizaje independiente, así como para reforzar la calidad didáctica en el aula.

- Existen carencias metodológicas para involucrar más a estos estudiantes en el aprendizaje del inglés, darle más confianza porque tienen temor a hablar, demostrado por su poca participación.

- Algunos estudiantes refugiados tienen muy bajo nivel en la lengua madre, lo que dificulta la comprensión de contenidos importantes del inglés.

La técnica de observación de las clases utilizada por la docente responsable del proyecto de vinculación reveló un aprendizaje lento por parte de los estudiantes refugiados; sin embargo, estos fueron capaces de vencer los objetivos instructivos de cada unidad didáctica. Se reveló el deseo de aprender de los estudiantes, a pesar de que tenían temor a cometer errores y a quedar chasqueados. Los estudiantes- docentes utilizaron métodos activos que ayudaron en el aprendizaje, pero carecían de algunos materiales didácticos que ayudaran a los estudiantes a desarrollar el trabajo independiente, tanto en el aula como en la casa.

Se estableció un nexo fraternal y de camaradería entre los estudiantes-docentes y los refugiados en el proceso de enseñanza-aprendizaje, logrando que la mayoría de los estudiantes refugiados fueran capaces de decir el abecedario, los números, preguntar la hora y darla, saludarse, presentarse, responder preguntas vitales para entrevistas laborales, en un mes y medio de clases. En algunos estudiantes esto no se obtuvo con facilidad, especialmente en los que eran analfabetos en su propia lengua o con un nivel bajo de escolaridad, coincidiendo con los resultados de Black (2013) en sus investigaciones. Fue imprescindible reciclar los contenidos en el aula en la misma clase y en diferentes clases hasta que los estudiantes lograran aprender, lo que también reveló Kaur (2011) en su estudio. Los logros en el aprendizaje después del reciclaje hicieron que los estudiantes 
refugiados se sintieran más seguros en el proceso de enseñanza-aprendizaje y esto los motivó a proseguir a una nueva etapa con más motivación y confianza.

Los elementos observados permitieron a la responsable del proyecto asesorar a los estudiantes- docentes encargados de las clases, en la reestructuración de las actividades docentes, uso de una metodología adecuada y de vincular la dimensión afectiva con la cognitiva y la praxiológica en este proceso a una población tan importante y vulnerable.

Al aplicar la encuesta a los estudiantes refugiados se reveló (Gráfico 1) que el 53\% de ellos eran emprendedores, y el $47 \%$ pertenecían al grupo de reasentamiento en un país de habla inglesa. Se aprecia que el menor porcentaje está representado por los refugiados que se van a reasentar en otro territorio. Esto podría estar causado por falta de interés, motivación, poca conciencia de la necesidad de conocer el inglés para su futuro, entre otras causas. Se distingue al grupo de emprendedores como de más mayoría de participación en el curso de inglés, más conscientes de la necesidad de mejorar en su negocio y aprecian en el inglés una posibilidad de optimizar su empresa.

Gráfico 1. Cantidad de refugiados del grupo de emprendedores y del grupo de reasentamiento

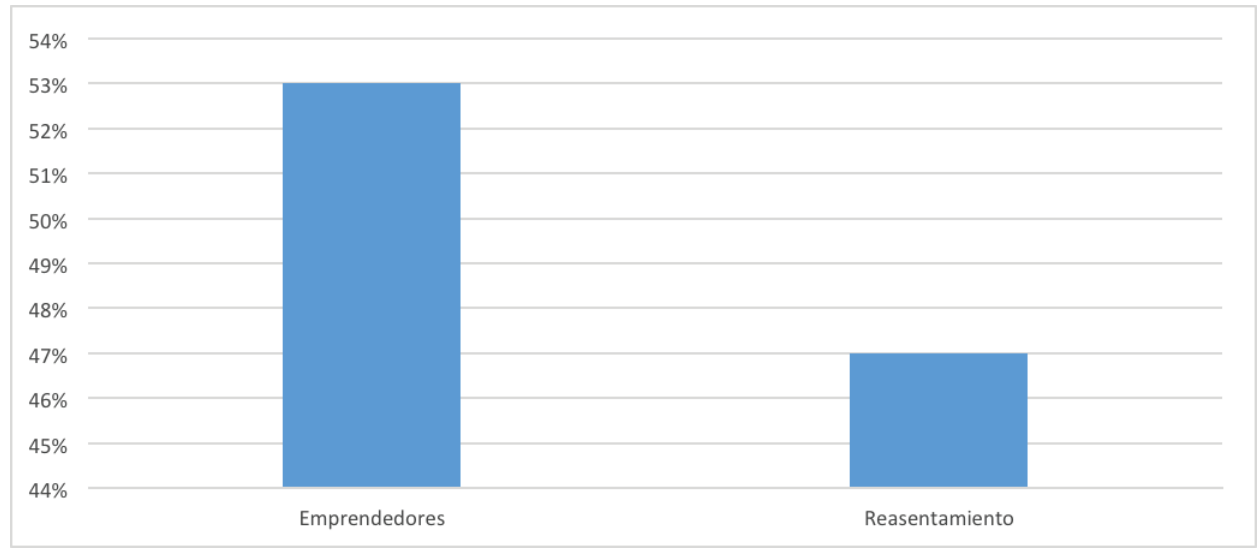

Fuente: encuesta. Elaboración propia

Cuando se analizan los emprendimientos más frecuentes (Gráfico 2), llama la atención cómo el predominio está en las ventas, y dentro de ellas, las ventas de ropa, seguidas por venta de celulares y de alimentos, en igual proporción. Esto demanda la enseñanza de terminología basada en estas actividades y las funciones comunicativas relativas a las ventas. 
Gráfico 2. Actividades que realizan los emprendedores como sostenibilidad

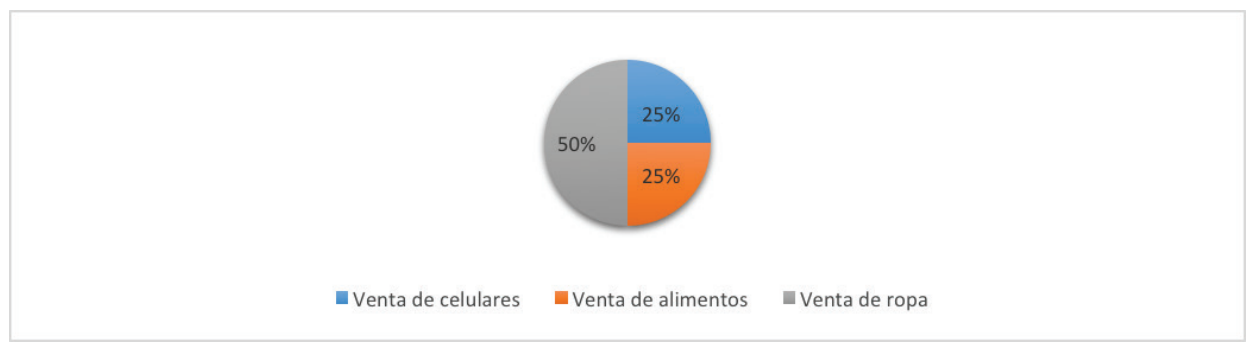

Fuente: encuesta. Elaboración propia

Con relación a las necesidades de aprendizaje, los estudiantes refugiados revelaron, como se aprecia en el Gráfico 3, que tienen necesidad de comunicarse en idioma inglés para cumplir funciones comunicativas elementales en idioma inglés, como lo es dar y solicitar direcciones, deletrear su nombre, saludar, así como brindar información personal, lo que se asocia a sus necesidades vitales de comunicación para una entrevista de trabajo. Con relación a sus oficios, muestran que necesitan aprender inglés para ordenar comida en un restaurante y comprar, así como solicitar trabajo.

Lo anterior demuestra que hay demanda de enseñar el inglés vinculado a las ocupaciones de los empleadores, porque la mayoría de ellos, como se apunta con anterioridad, eran vendedores. Con relación al aprendizaje de elementos necesarios para la comunicación se coincide con Adkins, et al, (1999), los que plantean reducir el estrés de los estudiantes refugiados enseñándoles funciones comunicativas vitales, que coinciden con las encontradas en esta encuesta.

Gráfico 3. Necesidades para las que los que los refugiados necesitan aprender el idioma inglés

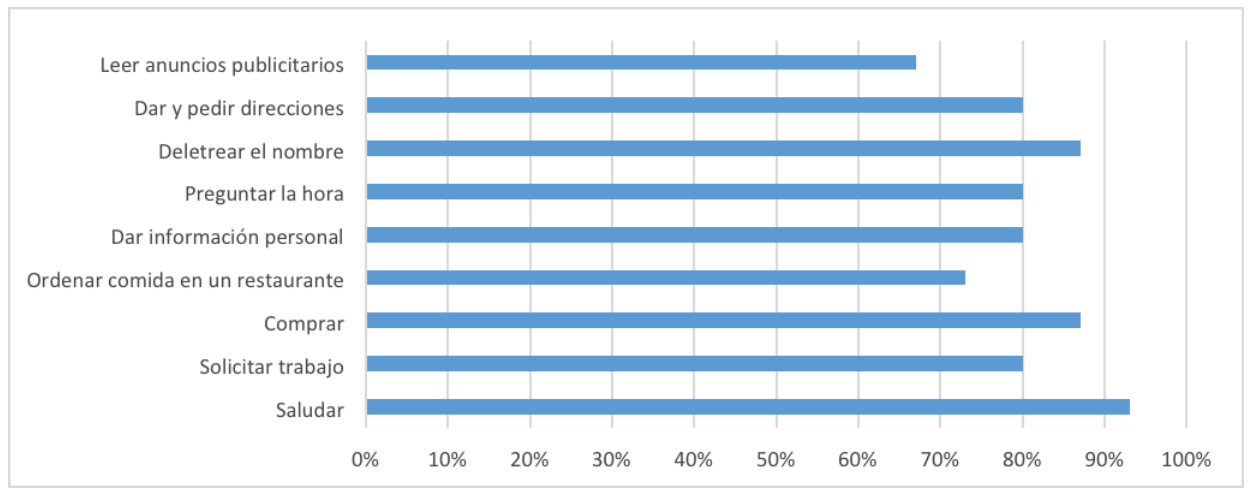

Fuente: encuesta. Elaboración propia 
Al ser cuestionados sobre otras necesidades de aprender el idioma inglés, según su interés, un elevado porcentaje de estudiantes refugiados reflejó, entre otras necesidades, el ver la televisión (Gráfico IV). Analizando su futuro reasentamiento en un contexto de habla inglesa, se aprecia cómo algunos encuestados refirió la necesidad de aprender vocabulario relacionado con ir al médico y dialogar de manera fluida, entre otras necesidades y, en menor escala, el visitar al médico, tomar medicamentos, comprarlos, mencionar las partes del cuerpo, arrendar vivienda y ayudar a sus hijos.

Gráfico 4. Otras necesidades para las que los que los refugiados necesitan aprender el idioma inglés

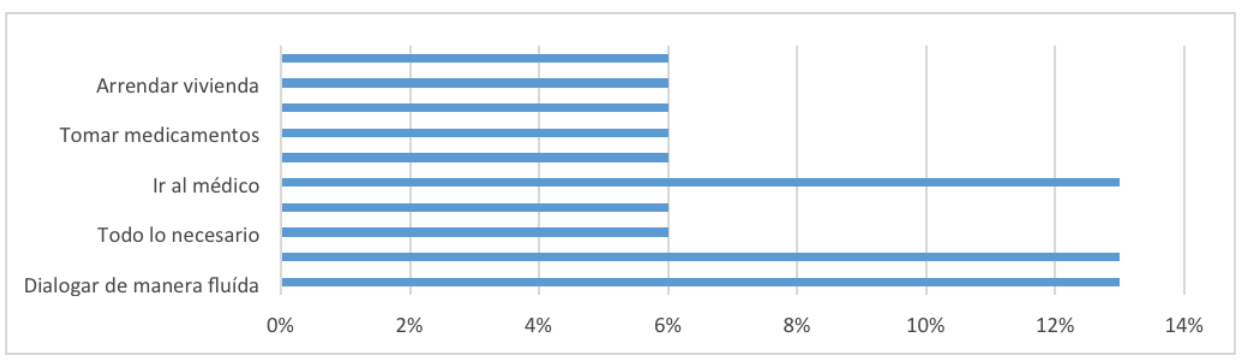

Fuente: encuesta. Elaboración propia

En el aspecto sobre las formas en las que les gustaría aprender el idioma inglés (Gráfico V) se muestra que el mayor porcentaje (80\%) tiene predilección por las parejas y grupos, es decir, aprender en comunicación con otros. También el mismo porciento se muestra interesado en aprender el idioma inglés a través de explicaciones, lo cual no quiere decir que les agrada aprender de una forma tradicional, sino que puede significar que, aparte de aprender en grupos y parejas, sería factible recibir una explicación clara de los contenidos que se imparten.

Otro porciento significativo expresó su agrado de aprender a través de juegos y canciones, es decir, con una metodología lúdica que les permita un aprendizaje flexible. Es importante tener en cuenta que los estudiantes refugiados, en su mayoría, revelan ansiedad y estrés debido a problemas causados por encontrase en un contexto extraño, con una cultura diferente, y por el hecho de haber sido perseguidos de una u otra manera. También sienten agrado por el aprendizaje basado en diálogos. Solamente un 33\% siente el gusto por aprender de manera individual, lo que denota falta de agrado por la socialización, lo que podría tratarse de rechazo a la involucración social, algo común en estudiantes refugiados. 
Gráfico 5. Formas en las que a los refugiados les gustaría aprender el idioma inglés

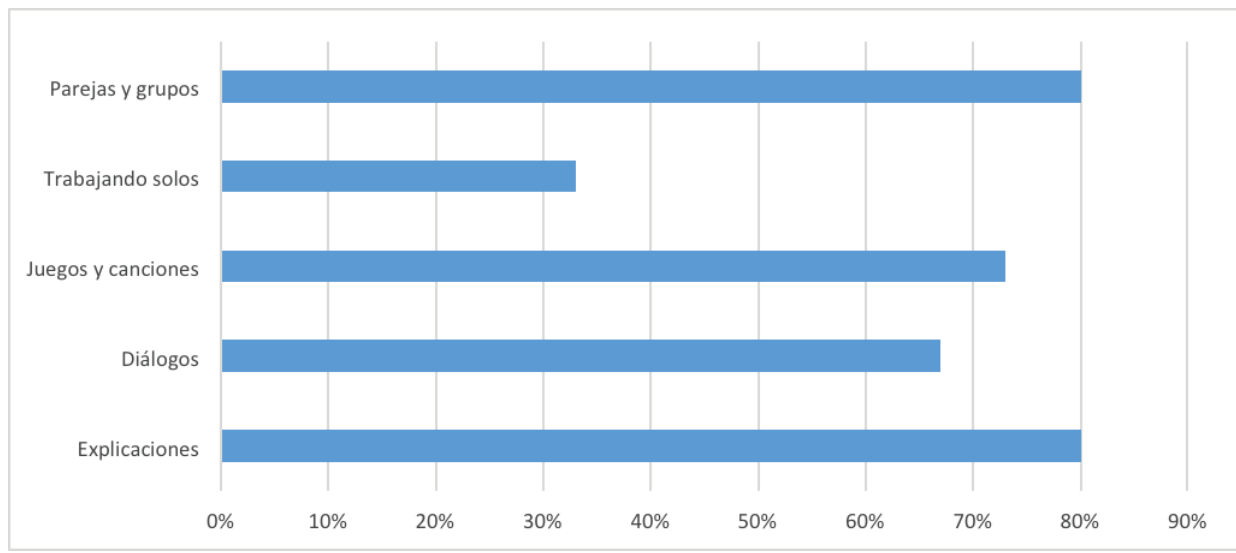

Fuente: encuesta. Elaboración propia

En lo concerniente a materiales didácticos para su aprendizaje independiente en la casa, (Gráfico 6), la mayoría de los estudiantes refugiados reveló la necesidad de cuadernos, seguido por libros, lápices de colores y videos, y luego por folletos, entre otros. Esto demuestra que para los cursos de idioma inglés para estudiantes refugiados se necesita el diseño de materiales que les facilite el trabajo desde la casa, de manera independiente. Se coincide con Hernández y Villalba (2005) citando a Cabré al enfatizar la necesidad de capacitar a los estudiantes y para ello diseñar materiales didácticos esenciales. Estos, como plantea Gass (1997), sirven para vincular la escuela con el hogar.

Gráfico 6. Materiales didácticos que desearían tener para estudiar de manera independiente

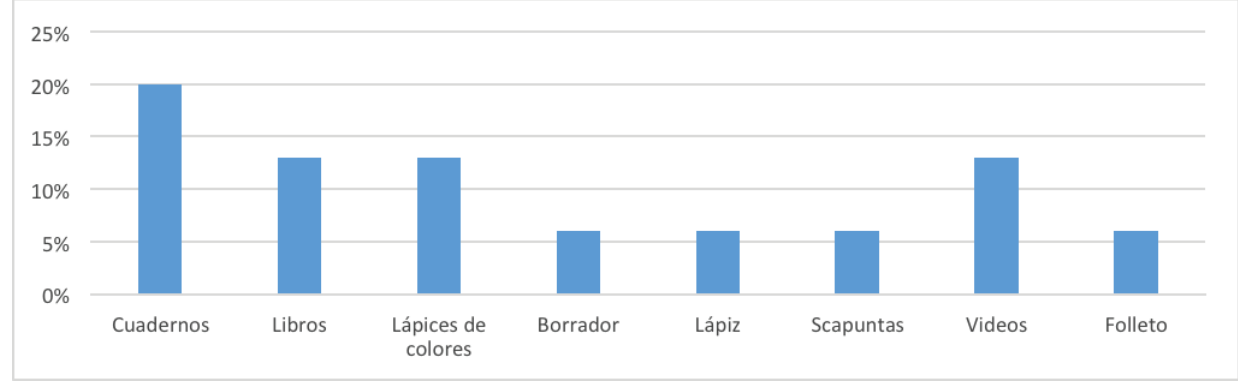

Fuente: encuesta. Elaboración propia

Al triangular los resultados obtenidos en las tres técnicas utilizadas se pudo constatar la necesidad de estructurar un proceso pedagógico para estudiantes refugiados con las dimensiones afectiva, cognitiva y praxiológica bien relacionadas, con contenidos basados 
en las necesidades de comunicación de acuerdo al emprendimiento actual y futuro, y a la necesidad de sobrevivencia en el reasentamiento en países de habla inglesa. Para ello es necesaria la elaboración de materiales didácticos y procedimientos metodológicos bien específicos basados en el diagnóstico realizado.

\section{CONCLUSIONES}

Los estudiantes refugiados necesitan un proceso pedagógico que tenga en cuenta la didáctica del inglés orientada a sus necesidades de comunicación, con materiales didácticos diseñados para este fin, los que pueden variar entre folletos o libros con las estructuras fundamentales, funciones comunicativas y el léxico de acuerdo a sus emprendimientos actuales o futuros y a la sobrevivencia. Además, es crucial desarrollar un proceso educativo humanista, histórico-cultural y liberador, basado en la inclusión, seguridad, amor, libertad para lograr el aprendizaje.

La puesta en práctica de un modelo pedagógico que combine las dimensiones afectiva, cognitiva y praxiológica para el aprendizaje del idioma inglés puede mejorar el rendimiento de los refugiados, al tener en cuenta no solo los contenidos, sino los sentimientos y necesidades de los mismos como población vulnerable, la que demanda inclusión a la sociedad y a la universidad a través de proyectos de vinculación.

Se propone una metodología que, desde las dimensiones apuntadas, permita un clima de libertad en el aula, donde los estudiantes se sientan felices de hablar sobre su cultura, familia, experiencias vividas, sin la expectativa de ser criticados, más bien empoderarlos en su papel protagónico como aprendices y de quienes también se puede aprender en el intercambio social.

\section{PROPUESTA METODOLÓGICA}

Esta metodología consta de tres etapas con procedimientos para enseñar a refugiados el inglés con fines ocupacionales y de sobrevivencia desde las dimensiones cognitiva, afectiva y praxiológica con un enfoque comunicativo, humanista, histórico-cultural y liberador.

ETAPA 1. Eres libre para aprender

Objetivo: proveer a los estudiantes de un clima liberador para el aprendizaje

Procedimiento 1:

Presentaciones (conversaciones estudiantes-estudiantes y estudiantes-docentes)

Los estudiantes y docentes se presentan y tienen un intercambio diciendo los nombres, y enfatizando frases de complacencia por conocerlos. Esto se puede desarrollar en la lengua madre de los estudiantes. También el docente puede presentar las frases en inglés, hacer que los estudiantes repitan y luego usarlas en el aula en una conversación en parejas y grupos. 
Las funciones comunicativas principales serán:

Introducing yourself/ meeting people

My name is......./ What's your name?

Nice/Pleased to meet you

Nice/Pleased to meet you too

Luego se puede introducir la función comunicativa:

Requesting information about origin

I am from .....Where are you from?

I am from ......

Nice/Pleased to meet you!

Nice/Pleased to meet you too

Se enfatizarán las expresiones de bienvenida: Welcome to the class

\section{Procedimiento 2}

\section{Sistematización}

Consiste en la sistematización de las funciones comunicativas y léxico utilizado para las presentaciones a través de la ampliación del diálogo. Cada una de las partes practicadas se va a unir para formar un diálogo más amplio. A través de la sistematización los estudiantes podrán sentir éxito en el aprendizaje, al permitirles aprender y solidificar lo que aprende. No finalizar hasta que cada estudiante converse con otro y con el docente.

Al terminar esta etapa, los estudiantes refugiados sentirán que son bienvenidos, felices en relaciones de amistad, y que pueden aprender en un ambiente relajado y flexible.

\section{ETAPA II. Lo que necesitas aprender}

Objetivo: diagnosticar las necesidades de aprendizaje de los estudiantes

\section{Procedimiento 1}

Aplicar el test diagnóstico (encuesta) en la lengua madre. Para ello el docente debe dar las instrucciones necesarias a los estudiantes. En caso de estudiantes que tengan dificultad para escribir o leer en su idioma (en este caso el Español), el docente les leerá las preguntas y les completará las respuesta que ellos den (Esto ocurre en presencia de alumnos analfabetos, lo que puede ser posible). El docente no debe mostrar ninguna alarma en este caso, solo debe ayudar al estudiante con amabilidad.

En la parte de la encuesta donde se pide a los estudiantes que opinen, se debe lograr que los mismos se expresen con total libertad.

\section{Procedimiento 2}

Evaluar las encuestas. El docente calificará las respuestas de los estudiantes y evaluará sus necesidades de aprendizaje para diseñar las actividades docentes. 
ETAPA III. Diseño de lo que vas a aprender

Objetivo: diseñar las actividades docentes de acuerdo a las necesidades de aprendizaje diagnosticadas

\section{Procedimiento 1}

Realizar un listado de los contenidos que se incluirán en cada unidad didáctica

\section{Procedimiento 2}

De acuerdo a los contenidos organizar los demás componentes del proceso didáctico: objetivos, métodos, medios, forma de organización de la enseñanza y evaluación.

Establecer el papel del docente y de los estudiantes en cada una de las unidades didácticas.

Dar participación a los estudiantes en la elaboración de algunos materiales didácticos, como láminas, objetos, así participarán activamente del proceso de enseñanza-aprendizaje. Este protagonismo los empodera del proceso y les hace sentir éxito, lo que beneficia el aprendizaje.

\section{ETAPA IV. Ya aprendes}

Objetivo: impartir las actividades docentes

\section{Procedimiento 1}

Despertar el interés de los estudiantes con el uso de warm-ups llamativos a través de actividades lúdicas como juegos, música, videos y comentarios, poesía.

\section{Procedimiento 2}

Introducir el nuevo contenido a través de actividades simuladas a situaciones de la vida real. Involucrar a todos los estudiantes en la provisión de elementos de la lengua. El docente debe apoyarse en estudiantes que sí tienen algún estudio previo del idioma inglés, por lo que la imitación se convertirá en elemento potencial para aprender.

Mantener un clima relajado, flexible, de confianza, con música de fondo, láminas, colores, que hagan sentir felicidad en el momento en que se aprende.

El docente debe mantener la sonrisa, usar frases amables y de seguridad. Mientras el alumno trabaja en intercambio con otro, el docente monitorea la actividad y con paciencia hace sugerencias y corrige los errores. Cuando ya los estudiantes están presentando lo aprendido el docente no debe interrumpirlos para hacer correcciones, las que hará de manera general en otro momento, al final de la clase o en un turno extra de clases.

\section{REFERENCIAS BIBLIOGRÁFICAS}

Adkins, M. Birman, D. \& Sample, B. (1999). Cultural adjustment, mental health, and ESL: The refugee experience, the role of the teacher, and ESL activities. Denver, CO: Spring Institute for International Studies. Recuperado el 2 de diciembre de 2016 desde http://www.springinstitute. 
org/wp-content/uploads/2016/04/culturaladjustmentmentalhealthandesl2.pdf

Asamblea General de las Naciones Unidas. Proyecto de documento final de la cumbre de las Naciones Unidas para la aprobación de la agenda para el desarrollo después de 2015. Recuperado el 12 de noviembre de 2016 desde http://www.un.org/es/comun/docs/?symbol=A/69/L.85

Black, R. (2013) "Educating Ana: a retrospective diary study of pre-literate refugee students". EWU Master's Thesis Collection. Paper 84. Recuperado el 13 de diciembre de 2016 desde http:// dc.ewu.edu/cgi/viewcontent.cgi?article $=1083 \&$ context=theses

Domínguez, L. (2013). Perspectivas del enfoque histórico cultural para la psicología del desarrollo. Revista Amazônica, año 6, vol xi, (2)169-259. Recuperado el 27 de diciembre de 2016 desde https://dialnet.unirioja.es/servlet/articulo?codigo $=4730567$

Freire, P. (s.f). Pedagogía del Oprimido. Recuperado el 20 de diciembre de 2016 desde http://www. servicioskoinonia.org/biblioteca/general/FreirePedagogiadelOprimido.pdf

Gass, M. (1997). Input, interaction, and the second language learner, Mahwah, NJ:Lawrence Erlbaum associates. Recuperado el 20 de septiembre de 2016 desde de http://tesl-ej.org/ej09/ r14.html

Hernández, M. y Villalba, F. (2005). La enseñanza de Español con fines laborales para inmigrantes. Glosas Didácticas. Revista Electrónica Internacional. (5), 74-83. Recuperado el 15 de septiembre de 2016 desde http://diarium.usal.es/sazpiazu/files/2015/10/gd15-081.pdf

Hooks, B. (2003). Teaching Community: A Pedagogy of Hope, New York: Routledge. Recuperado el 18 de diciembre de 2016 desde file:///C:/Users/hramirez/Downloads/12009-37403-1-PB.pdf

Hutchinson, T. \& Waters, A. (1994). English for Specific Purposes, Cambridge: Cambridge University Press.

Hymes, D. (1970). On communicative competence. In Gumperz, J. and Hymes, D (eds.) Directions in Sociolinguistics (23-30). Holt, Rhinehart and Winson, New York.

Kaur, T. (2011). Personal Observations of English Learning in a Refugee Classroom. English Language Partners Hutt. Recuperado el 8 de noviembre de 2016 desde https://akoaotearoa. ac.nz/ako-hub/ako-aotearoa-central-hub/news/personal-observations-english-learning-refugeeclassroom

Munthe, M. (2005) An Analysis of the Language Challenges faced by Myanmar Refugee Students in Norway. Masther's Thesis. University of Stavanger, Canada. Recuperado el 10 de noviembre de 2016 desde https://brage.bibsys.no/xmlui/bitstream/handle/11250/185737/Munthe,\%20Myat. pdf?sequence $=1$

Richards, J. \& Rodgers, T. (1986). Approaches and Methods in Language Teaching, Cambridge: Cambridge University Press. Recuperado el 22 de noviembre de 2016 desde https://www. academia.edu/5666615/Approaches_and_Methods_in_Language_Teaching_-_Jack_C._ Richards_and_Theodore_S._Rodgers

Williams, M. \& Burden, R. (2005). Psychology for Language Teachers A social constructivist approach, Cambridge: Cambridge University Press. 
